\title{
Social influence on the effectiveness of virtual fencing in sheep
}

\author{
Danila Marini $^{\text {Corresp., } 1,2}$, Tellisa Kearton ${ }^{1,2}$, Jackie Ouzman ${ }^{3}$, Rick Llewellyn ${ }^{3}$, Sue Belson ${ }^{2}$, Caroline Lee $^{1,2}$ \\ ${ }^{1}$ School of Environmental and Rural Science, University of New England, Armidale, New South Wales, Australia \\ 2 Agriculture and Food, Commonwealth Scientific and Industrial Research Organisation, Armidale, New South Wales, Australia \\ 3 Agriculture and Food, Commonwealth Scientific and Industrial Research Organisation, Adelaide, South Australia, Australia \\ Corresponding Author: Danila Marini \\ Email address: danila.marini@csiro.au
}

Early virtual fencing trials have effectively contained small groups of sheep within set areas of a paddock when all animals were wearing manual electronic collars. With sheep farming commonly involving large flocks, a potential cost-effective application of virtual fencing would involve applying equipment to only a proportion of the flock. In this study, we tested the ability of virtual fencing to control a small flock of sheep with differing proportions of the group exposed to the virtual fence (VF). Thirty-six Merino sheep were identified as leaders, middle or followers by moving them through a laneway. The sheep were then allocated to groups balanced for order of movement. The groups ( $n=9$ per group) included applying the VF to the following proportions of animals within each group: 1) $100 \%(n=9$ VF) 2) $66 \%(n=6$ VF; $n=3$ no VF) 3) $33 \%$ ( $n=3$ VF; $n=6$ no VF) 4$) 0 \%$ (no VF; free to roam the paddock). The groups were given access to their own paddock ( 80 $x 20 \mathrm{~m}$ ) for two consecutive days, six hours per day, with the VF groups prevented from entering an exclusion zone that covered $50 \%$ of the north side of the paddock. During these hours, VF interactions, behavioural time budgets, and body temperature were recorded as measures of stress, and location was tracked with GPS. Group $100 \%$ VF and Control were tested on the first two days and groups $33 \%$ VF and $66 \%$ VF were tested on the following two days. During VF implementation the $100 \%$ VF and $66 \%$ VF group were successfully prevented from entering the exclusion zone. Having only $33 \%$ of the flock exposed to the virtual fence was not successful, with the sheep pushing forward through the $\mathrm{VF}$ to join flock mates in the exclusion zone. For learning to respond to the audio cue, sheep in the $33 \%$ group received more electrical stimuli with a 0.51 proportion for the ratio of electrical stimuli to audio cue, compared to 0.22 and 0.28 for the $100 \%$ and $66 \%$ groups, respectively. There were small differences in behavioural patterns of standing and lying on both days of testing, with the $100 \%$ VF and $66 \%$ VF groups spending more time lying. Although stress-induced hyperthermia did not occur in any of the VF groups, body 
temperature differed in the $33 \%$ VF group. There were no differences in temperature measures between the control and $100 \%$ VF animals. This study demonstrates that for a short period, controlling two-thirds of the flock was equally as effective as virtually fencing all animals, while controlling one-third of a flock with a virtual fence was not effective. For the short term, it appears that implementing the VF to a proportion of the flock can be an effective method of containment. Due to the limitations of this study, these results warrant further testing with larger flocks and for longer periods. 


\section{Social influence on the effectiveness of virtual fencing}

2 in sheep

3

4

5

6

7

8 9

14

15

16

17

21

22

23

24

25

26

27

28

29

30

31

32

33

34

35

36

37
Danila Marini ${ }^{1,2}$, Tellisa Kearton, ${ }^{1,2}$, Jackie Ouzman $^{3}$, Rick Llewellyn ${ }^{3}$, Sue Belson ${ }^{2}$, Caroline Lee ${ }^{1,2}$

${ }^{1}$ School of Environmental and Rural Science, University of New England, Armidale, NSW, Australia

${ }^{2}$ Agriculture and Food, Commonwealth Scientific and Industrial Research Organisation, Armidale New South Wales, Australia

${ }^{3}$ Agriculture and Food, Commonwealth Scientific and Industrial Research Organisation, Adelaide, South Australia, Australia

Corresponding Author:

Danila Marini ${ }^{1,3}$

Building W77, School of Environmental and Rural Science, University of New England, Armidale, New South Wales, 2350, Australia

Email address: danila.marini@csiro.au 


\section{Abstract}

39

40

41

42

43

44

45

46

47

48

49

50

51

52

53

54

55

56

57

58

59

60

61

62

63

64

65

66

67

68

69

70

71

72

73

74

75

76

77
Early virtual fencing trials have effectively contained small groups of sheep within set areas of a paddock when all animals were wearing manual electronic collars. With sheep farming commonly involving large flocks, a potential cost-effective application of virtual fencing would involve applying equipment to only a proportion of the flock. In this study, we tested the ability of virtual fencing to control a small flock of sheep with differing proportions of the group exposed to the virtual fence (VF). Thirty-six Merino sheep were identified as leaders, middle or followers by moving them through a laneway. The sheep were then allocated to groups balanced for order of movement. The groups ( $\mathrm{n}=9$ per group) included applying the VF to the following proportions of animals within each group: 1) $100 \%(n=9 \mathrm{VF}) 2$ ) $66 \%(n=6 \mathrm{VF} ; \mathrm{n}=3$ no VF) 3) $33 \%(n=3 \mathrm{VF} ; n=6$ no VF) 4) $0 \%$ (no VF; free to roam the paddock). The groups were given access to their own paddock $(80 \times 20 \mathrm{~m})$ for two consecutive days, six hours per day, with the VF groups prevented from entering an exclusion zone that covered $50 \%$ of the north side of the paddock. During these hours, VF interactions, behavioural time budgets, and body temperature were recorded as measures of stress, and location was tracked with GPS. Group 100 $\% \mathrm{VF}$ and Control were tested on the first two days and groups $33 \% \mathrm{VF}$ and $66 \% \mathrm{VF}$ were tested on the following two days. During VF implementation the $100 \% \mathrm{VF}$ and $66 \% \mathrm{VF}$ group were successfully prevented from entering the exclusion zone. Having only $33 \%$ of the flock exposed to the virtual fence was not successful, with the sheep pushing forward through the VF to join flock mates in the exclusion zone. For learning to respond to the audio cue, sheep in the $33 \%$ group received more electrical stimuli with a 0.51 proportion for the ratio of electrical stimuli to audio cue, compared to 0.22 and 0.28 for the $100 \%$ and $66 \%$ groups, respectively. There were small differences in behavioural patterns of standing and lying on both days of testing, with the $100 \% \mathrm{VF}$ and $66 \%$ VF groups spending more time lying. Although stressinduced hyperthermia did not occur in any of the VF groups, body temperature differed in the 33 $\% \mathrm{VF}$ group. There were no differences in temperature measures between the control and $100 \%$ VF animals. This study demonstrates that for a short period, controlling two-thirds of the flock was equally as effective as virtually fencing all animals, while controlling one-third of a flock with a virtual fence was not effective. For the short term, it appears that implementing the VF to a proportion of the flock can be an effective method of containment. Due to the limitations of this study, these results warrant further testing with larger flocks and for longer periods. 


\section{Introduction}

79

80

81

82

83

84

85

86

87

88

89

90

91

92

93

94

95

96

97

98

99

100

101

102

103

104

105

106

107

108

109

110

111

112

113

114

115

116

117

Virtual fencing is an emerging technology with the ability to contain livestock within a boundary through the implementation of a warning cue (audio tone) and a negative stimulus (electrical stimulus). Through associative learning animals' pair an audio warning with an electrical stimulus and quickly learn to avoid the virtual fence by responding to the audio warning alone (Campbell et al. 2018; Marini et al. 2018b). Virtual fencing for cattle currently works on the premise that all animals require the virtual fence to be implemented, however this method may not be economically feasible in the Australian sheep industry due to the large size of many flocks. The efficacy of virtually fencing and controlling only a proportion of sheep in a flock using collars could provide an opportunity to reduce costs.

Currently, there is no commercially available virtual fencing system for sheep. Virtual fencing in sheep has been implemented experimentally using commercially available dog collars but as a new technology, it is important to measure the impact of virtual fencing on sheep welfare, particularly in situations that may affect flock interactions. Initial studies in sheep have shown that they readily learn to respond to an audio cue to avoid an electrical stimulus when trained individually (Marini et al. 2019; Marini et al. 2018a), a fundamental component required for virtual fencing to be ethically acceptable (Lee et al. 2018). There have been few studies that have evaluated group responses to the virtual fence and whether a group of sheep could be trained in a paddock environment (Brunberg et al. 2017; Jouven et al. 2012). These studies found mixed success in containing animals within a paddock. In a controlled grazing study using a manually implemented virtual fence with a small flock of six naïve sheep, (Marini et al. 2018a) numerous group interactions were observed where the flock approached the virtual fence line together, but only one individual received the VF cues but all of the animals in the group reacted. This suggests that there are social influences acting within a group when virtual fencing is implemented. Indeed, recently, social facilitation of learning virtual fencing responses were demonstrated in beef cattle (Keshavarzi et al. 2020).

Sheep flock movements are a continuous process of individual and group decisions that influence how a group reacts and moves (Ramseyer et al. 2009a). Sheep flock movements are affected by a variety of factors, including the flocks' activity, size, food availability, age, breed and social relationships within the flock (Hauschildt \& Gerken 2016; Pillot et al. 2010; Ramseyer et al. 2009b; Taylor et al. 2011). With the strong flocking behaviour and behaviour synchronisation of sheep (Hauschildt \& Gerken 2015; Hauschildt \& Gerken 2016), there may be potential to only have a proportion of sheep controlled with the virtual fence and still gain effective control (Taylor et al. 2011). However, the proportion of trained to naïve individuals can impact the response of the flock, as observed by Jouven et al. (2012), where a flock with a larger proportion of sheep without the virtual fence resulted in ineffective containment within the virtual fence.

Behavioural time budgets have been used previously in cattle (Campbell et al. 2017; Campbell et al. 2019) and sheep (Marini et al. 2018a) exposed to virtual fencing, as indicators of 
118 impact to welfare through behavioural change. Another method of measuring impact on welfare

119

120

121

122

123

124

125

126

127

128

129

130

131

132

133

134

135

136

137

138

139

140

141

142

143

144

145

146

147

148

149

150

151

152

153

154

155

156

157

includes measures of body temperature. Stress induced hyperthermia as measured by an increased body temperature has been previously shown to be an indicator of stress and anxiety states in sheep (Pedernera-Romano et al. 2010; Sanger et al. 2011). These measures will be used to determine the welfare of the animals in the different treatment groups. (Hauschildt \& Gerken 2015; Hauschildt \& Gerken 2016; Taylor et al. 2011).

In this study we tested the ability for virtual fencing to control a small flock of sheep when differing proportions of the group were exposed to the virtual fence (VF). The hypotheses being tested are 1) the virtual fence would be effective at containing a small flock when $66 \%$ of animals had an active virtual fence 2) the flock with $66 \%$ of animals exposed to the virtual fence would be more effective than $33 \%$ exposed and 3 ) we predict sheep in the $33 \%$ group would have altered behavioural time budgets and increased body temperature, but not the $66 \%$ and $100 \%$, compared to control sheep. The aim of this study was to determine if virtually fencing differing proportions of sheep in a flock would affect the efficacy of containment within a virtual fence, as well as determine effects on sheep behaviour and body temperature as indicators of animal welfare.

\section{Materials \& Methods}

\section{Ethical Statement}

The protocol and conduct of the study were approved by the CSIRO McMaster Laboratory Animal Ethics Committee under the New South Wales Animal Research Act 1985 (approval ARA 17/24). The trial was conducted at Waikerie, South Australia, in the Autumn of 2018. Average minimum temperature was $10.5^{\circ} \mathrm{C}$ and maximum temperature was $31.5^{\circ} \mathrm{C}$, with windspeeds between 22-33 kmph.

\section{Animals}

Thirty-six Merino ewes $(41.6 \pm 0.62 \mathrm{~kg})$ were used in the study. The sheep were marked for visual identification from 1 to 36 using wool paint (Dy-Mark, Australia) placed on the back and either side of the barrel. Sheep were shorn the week prior to testing and required the wool around their neck to be reclipped using handheld shears on the first test day. During the day sheep were kept on a forage barley crop which was grown in the trial paddocks; at night they were moved to holding yards and supplemented with hay (approximately $1 \mathrm{~kg}$ each). Water was provided ab libitum in the inclusion zone of the experimental paddock and in the holding yards. Sheep were kept and moved within their experimental groups throughout the trial.

\section{Equipment}

The virtual fence was implemented with Garmin dog training equipment. This included a GPS track and train collar (Garmin TT15, Garmin Ltd, Olathe, KS, USA) and GPS hand-held unit (Garmin Alpha 100, Garmin Ltd, Olathe, KS, USA). The collars administer an audible sound (70

Peer) reviewing PDF | (2020:05:48487:2:0:NEW 2 Sep 2020) 
158

159

160

161

162

163

164

165

166

167

168

169

170

171

172

173

174

175

176

177

178

179

180

181

182

183

184

185

186

187

188

189

190

191

192

193

194

195

196

197

$-80 \mathrm{~dB}, 2.7 \mathrm{kHz})$. The electrical stimulus was set to level $4(36 \mathrm{~mA}, 20 \mu \mathrm{s}$ with 16 pulses delivered per s) as determined by previous studies (Marini et al 2018b). Each collar was paired to its own hand-held unit.

The day prior to the first test day, Thermachron iButton temperature loggers (Embedded Data Systems, Lawrenceburg, USA) were inserted into the vagina to measure core body temperature. These were attached to an EAZI-BREED CIDR ${ }^{\circledR}$ (Zoetis, New Jersey, USA). The CIDR's were leached of hormone in a phosphate-buffered saline (PBS) solution prior to use. They were assembled using heat shrink tubing as previously described (Fisher et al. 2008; Lea et al. 2008). Temperature was recorded at $1 \mathrm{~min}$ intervals for the duration of the trial. The loggers were removed at the completion of testing on day two for each group.

At the beginning of each test day the sheep were fitted with $\mathrm{HOBO} \otimes$ Pendant $\mathrm{G}$ accelerometers, (Onset Computer Corporation, Pocasset, MA, USA) to measure standing and lying behaviours. These were attached to the outside of the left hind leg mid-side on the cannon bone with a bandage and were removed at the end of each test day. The loggers recorded $\mathrm{x}, \mathrm{y}, \mathrm{x}$ coordinates that corresponded to a state of either standing/grazing, walking/running or lying, this was recorded every $2 \mathrm{~s}$ between the hours of 9:30 a.m. to 3:30 p.m. The sheep in this study have not previously worn the loggers.

\section{Test procedures}

The sheep were weighed, then identified as leaders, middle or followers prior to group allocation. Group movement order in sheep has been previously identified as stable and a way to categorise a sheep's position in a flock (Squires and Daws, 1975), with sheep at the back of the flock having a more stable order (Doughty and Hinch, 2014). To identify group order, the sheep were walked calmly down a lane way six times and visualized using a drone. Sheep that were identified in the front and back of the flock four times were classed as leaders and followers respectively, with eight sheep identified in each category. They were then allocated to group balanced for order, it was ensured that at least one sheep from each subgroup (leader, follower and middle) had the virtual fence implemented within each group i.e. in the $33 \%$ group one leader, one follower and one middle group animal had the VF implemented. The groups ( $n=9$ per group) were:

1) $\quad 100 \%$ of sheep with active virtual fence ( $\mathrm{n}=9$ virtual fence)

2) $66 \%$ of sheep with active virtual fence ( $n=6$ virtual fence; $n=3$ no virtual fence)

3) $33 \%$ of sheep with active virtual fence ( $n=3$ virtual fence; $n=6$ no virtual fence)

4) $0 \%$ of sheep with active virtual fence ( $\mathrm{n}=9$ no virtual fence); free to roam the paddock

Each group of sheep were tested in four separate paddocks $(80 \times 20 \mathrm{~m}$, Figure 1) that were as similar as possible. Test groups were visually isolated from each other by an opaque fence line 
198

199

200

201

202

203

204

205

206

207

208

209

210

211

212

213

214

215

216

217

218

219

220

221

222

223

224

225

226

227

228

229

230

231

232

233

234

235

236

using shade cloth. The flocks were given access to a paddock for two consecutive days for six hours per day. Group $100 \%$ and control were tested on the first two days and groups $33 \%$ and $66 \%$ were tested on the following two consecutive days between 8 am and $4 \mathrm{pm}$. Sheep in groups with the virtual fence were prevented from entering an exclusion zone that covered $50 \%$ of the paddock. Sheep were fitted with Garmin collars at the beginning of each test day. These collars were used to implement the virtual fence and track the sheep through GPS to monitor their location throughout the trial.

\section{Virtual fence implementation}

Prior to testing, a virtual fence and a warning zone (approximately $2 \mathrm{~m}$ apart) were discretely marked on the fence lines with coloured tape to ensure the collar operators were spatially consistent at delivering cues. When a sheep entered the warning zone, they were given an audio cue for $2 \mathrm{~s}$. If the sheep continued to walk forward or did not alter their behaviour (i.e. turn around or stop) after the audio cue, an immediate electrical stimulus $(<1 \mathrm{~s})$ was given. If the animal ran into the exclusion zone, the audio cues and stimuli were not applied. Once the animal was calm it was again given an audio cue and a stimulus if it proceeded further into the exclusion zone until it either ceased forward movement or began exiting the exclusion zone.

The operators of the Garmin collars were positioned on a viewing platform approximately $20 \mathrm{~m}$ outside the boundary of the test paddock. Each operator was in control of two sheep using two Garmin handheld GPS units. All sheep were collared but the collars of the uncontrolled sheep were not assigned to a handheld unit. Fence interactions were recorded by the operator using a voice recorder and scribing. All proceedings were videotaped. audio and electrical stimuli delivered were recorded during the trial and were later confirmed using video recordings. Interobserver agreement was assessed, agreement amongst the observers was $85 \%$.

\section{Statistics}

All data were analysed using the statistical software program $\mathrm{R}$ (The R Development Core Team Version 3.5.1.), the nlme (Pinheiro et al. 2018) package was used for analysis. Data were tested for normality through visual inspection of residual plots and the Shapiro-Wilks test $<0.05$ was considered statistically significant and $0.1>\mathrm{P}>0.05$ was considered a statistical tendency. The comparison of the audio cue and electrical stimulus applied to the groups was analysed using a linear model, data was not normal and required a square root transformation. Analysis of the cues across day within group was tested using a dependent t-test. The proportion of electrical stimuli to audio cues received by sheep in the virtual fence groups (number of audio cues was the number of interactions with the virtual fence) was analysed with a binomial proportions test.

Data collected from the hobo loggers were aggregated into 15 min intervals and further collated and presented as the proportion of total time spent in a behaviour for each day. Behaviour was analysed using a linear mixed effects model, fitting group, day and their interaction as a fixed 
237

238

239

240

241

242

243

244

245

246

247

248

249

250

251

252

253

254

255

256

257

258

259

260

261

262

263

264

265

266

267

268

269

270

271

272

273

274

275

276

effect, individual sheep was fitted as a random effect. The effects of cohort were accounted by the effect of group.

CircWave software (v 1.4, 2007 R. Hut) was used to perform a cosinor analysis in order to generate a $24 \mathrm{~h}$ Fourier curve periodogram for the body temperature data. The transformed fitted body temperature data was subsequently analysed for commonly used body temperature circadian wave parameters, these were: trough, peak, acrophase (the time at which peak occurs), range of oscillation, mesor (mean value of a wave), and amplitude (the difference between the peak and the mesor). Groups were compared with a one-way repeated measure analysis of variance (ANOVA) using a linear mixed effects model with individual sheep as a random effect. Due to data collection occurring across different days, the control group could only be compared with the $100 \%$ VF group, and the $33 \%$ VF group could only be compared to the $66 \%$ VF group for temperature data.

\section{GPS data}

GPS data from Garmin collars was collected for each trial day for each animal. Producing the residency grids, was undertaken in ArcGIS software suite (v9.2; ESRI, Redlands,CA, USA) using a suite of tools from the Hawth's Tools extension. The GPS data was interpolated onto a $30 \mathrm{~s}$ time step filling missing values with the previous known location, this was done for each animal per day. The trial paddock was divided into a vector grid of $2 \mathrm{~m}$ and the individual sheep data was overlaid. For each cell in the grid we summed the time spent and aggregated to produce a residency map for the flock over the trial duration.

\section{Results}

\section{Effectiveness of the virtual fence}

The effectiveness of excluding sheep from the exclusion zone was successful for $100 \% \mathrm{VF}$ and $66 \%$ VF groups but not the $33 \%$ VF group (Figure 2). Fence interactions were recorded for all groups except the control which did not have a fence implemented. Table 1 shows a summary of group interactions with the virtual fence line. There were differences between the groups (100\% $\mathrm{VF}, 66 \% \mathrm{VF}$ and $33 \% \mathrm{VF})$ and the proportion of electrical stimulus to audio cues given $\left(\chi^{2}(2)\right.$ $=13.3, \mathrm{P}<0.05)$ over the two days of testing. Proportion for the ratio of electrical stimulus to audio cue were 0.22 for the $100 \%$ VF group, 0.28 for the $66 \%$ VF group and 0.51 for the $33 \%$ VF group, indicating that the $33 \%$ VF group received a higher proportion of electrical stimuli. Interactions with the fence increased on day 2 as seen by the increase in audio cues delivered $\left(\mathrm{F}_{1}\right.$ $=10.7, \mathrm{P}<0.05)$. Two of the sheep with the virtual fence implemented in the $33 \% \mathrm{VF}$ group reached the maximum number (5) of electrical stimuli allowed to be given to an individual animal within one interaction with the fence. This meant that stimuli were ceased and led to the group crossing over the virtual fence and into the exclusion zone. Once in the exclusion zone the animals were not able to be turned back with the technology this led to the flock residing at the Northern fence line. 
278

279

280

281

282

283

284

285

286

287

288

289

290

291

292

293

294

295

296

297

298

299

300

301

302

303

304

305

306

307

308

309

310

311

312

313

314

315

316

\section{Behaviours}

Reactions to the virtual fence cues varied between the groups (Table 2 and 3 ). Sheep in the $33 \%$ group were the only ones to display negative behaviours in response to the cues, such as running forward in response to application of the audio cue.

For behavioural time budget, a group and day effect were seen $(\mathrm{P}<0.05$, Figure 3$)$ for lying $\left(F_{3,23}=7.7\right)$ standing $\left(F_{3,23}=6\right)$ and walking $\left(F_{3,23}=12\right)$. As standing is the opposite of lying, only the results of lying and walking behavior are presented. On day one there was no difference in time spent lying between the control group and the other groups. A difference in lying time was observed between the $100 \% \mathrm{VF}$ group and the $33 \% \mathrm{VF}$ group $\left(\mathrm{t}_{32}=-3.17, \mathrm{P}=0.003\right)$ with the $33 \%$ VF group spending $12 \%$ less time lying. On day two, differences in lying were observed between the $66 \% \mathrm{VF}($ mean $=28 \%)$ compared to the control $\left(\right.$ mean $=12 \%, \mathrm{t}_{32}=4.10$, $\mathrm{P}=0.003)$ and $100 \% \mathrm{VF}$ groups (mean $\left.=13 \%, \mathrm{t}_{32}=-3.7, \mathrm{P}=0.009\right)$. For walking behaviour, the $66 \%$ VF group spent significantly less time walking compared to the control and $100 \%$ group. On day one the $66 \% \mathrm{VF}$ group spent $2 \%$ of their time walking, the $100 \% \mathrm{VF}$ group spent $5 \%\left(\mathrm{t}_{32}=-3.50, \mathrm{P}=0.001\right)$. On day two the differences were observed between $66 \% \mathrm{VF}$ $($ mean $=2 \%)$ and control $\left(\right.$ mean $\left.=5 \%, \mathrm{t}_{32}=-8.64, \mathrm{P}<0.05\right)$ and $100 \% \mathrm{VF}\left(\right.$ mean $=6 \%, \mathrm{t}_{32}=-$ 10.61, $\mathrm{P}<0.05)$.

\section{Temperature}

For all the body temperature parameters measured, there were no significant differences between the control and $100 \% \mathrm{VF}$ group. The $33 \% \mathrm{v}$ group showed lower values for trough $\left(\mathrm{F}_{(3 / 30)}=\right.$ 4.700, $P=0.008)$ and acrophase $\left(\mathrm{F}_{(3 / 30)}=4.434 P=0.011\right)$ than the $66 \% \mathrm{VF}$ group, and higher values for range of oscillation $\left(\mathrm{F}_{(3 / 30)}=5.006, P=0.006\right)$ and amplitude $\left(\mathrm{F}_{(3 / 30)}=6.874, P=\right.$ 0.001 ) than the $66 \%$ VF group (Table 4).

\section{Discussion}

This study looked to determine if application of virtual fencing to differing proportions of sheep in a flock would affect the efficacy of containment during short term deployment. In the context of the current study, it appears that there is a minimum proportion of sheep that require virtual fencing to enable control of the entire group. Implementing the virtual fence on $66 \%$ of the flock was highly effective at containing a group of sheep and was similar to that reported in previous studies with small groups of sheep when $100 \%$ of the animals were virtually fenced (Jouven et al. 2012; Marini et al. 2018a). However, implementing the virtual fence on a smaller proportion of $33 \%$ of the flock was not effective at containing the flock within the virtual boundary. Further studies investigating proportions of animals controlled by the virtual fence in larger flock sizes are needed to test these finding over longer periods of time.

We observed some instances where sheep in the $66 \%$ VF group, that did not have the virtual fence implemented entered the exclusion zone, but they were encouraged back into the 
317 inclusion zone by the movements of the remaining flock. This demonstrates that having trained 318 individuals within a flock can be an effective method of controlling sheep movements. The

319

320

321

322

323

324

325

326

327

328

329

330

331

332

333

334

335

336

337

338

339

340

341

342

343

344

345

346

347

348

349

350

351

352

353

354

355

356 studies by Pillot et al. (2010); Taylor et al. (2011) trained sheep to respond to visual and auditory cues to approach a target (food reward), when these trained sheep were in a flock with naïve animals, they were successful in recruiting the naïve animals to approach the target when the cue was given, thereby influencing the flocks decision and movement patterns. For the animals with VF devices in the $33 \% \mathrm{VF}$ group, it appeared that their motivation to stay with their 'unfenced' flock mates when they entered the exclusion zone, was greater than the aversiveness of the electrical stimulus of the virtual fence. This was demonstrated by those sheep continuing to walk forward in response to the audio cue and electrical stimulus on several occasions to join their flock mates in the exclusion zone. The results seen in this study are similar to Jouven et al. (2012) where, during the 30 minutes of testing, they found naïve animals crossed over to the exclusion zone often but when they were in the minority they would return to the inclusion zone to re-join the rest of the flock. The precise social dynamics influencing the behaviour of virtually fenced sheep in groups has not yet been explored and future research in this area is needed, particularly for larger flock sizes more commonly found on commercial farms.

These findings should be considered in relation to the limitations of the study. These include the short period of the study ( 2 days of 8 hours per day per treatment), whereas over a longer period, social influences of the uncollared sheep may draw the collared sheep into the exclusion zone and reduce the effectiveness of containment of the sheep. Also, due to limitations on the number of collars and people available to administer the stimuli manually and the number of paddocks available only small group sizes could be tested, and treatments were not able to be replicated and tested on the same day. This brings in the potential for differences in environmental conditions that may have affected behaviour of the sheep in this study. With the development of automated collars, longer term larger scale replicated studies will be possible, as have been demonstrated in cattle (Campbell, et al. 2019).

The proportion of audio cue to electrical stimulus is an indicator of sheep learning to respond to the audio cue and avoid receiving the electrical stimulus. The lower the ratio, the better the learning shown by sheep. Within our study, the $33 \%$ VF group were the poorest learners with the highest ratio of electrical stimuli to audio cue at 0.50 . This ratio was similar to that seen in previous studies where sheep trained individually had a ratio of 0.48 (Marini et al. 2018b) and 0.51 (Marini et al. 2019). Whereas the $66 \%$ VF and 100\% VF had a similar mean ratio of 0.28 and 0.22 respectively, these results were more similar to studies that have trained animals in a group, where the mean application of electrical stimuli was 0.19 (Marini et al. 2018a). The mean number of electrical stimuli applied in the groups was also significantly different with sheep in the $33 \% \mathrm{VF}$ group receiving more electrical stimuli over the two days. This has implications for the welfare of the sheep in the $33 \% \mathrm{VF}$ group as animals may receive high numbers of stimuli, that could negatively impact their welfare. The sheep in the $33 \%$ group also displayed some negative behaviour in response to the cues such as running forward on the sound of the audio cue. Each group had an increase in audio cues and electrical stimuli applied 
357

358

359

360

361

362

363

364

365

366

367

368

369

370

371

372

373

374

375

376

377

378

379

380

381

382

383

384

385

386

387

388

389

390

391

392

393

394

395

on day two, although group approaches only increased slightly. Indicating there may have been more individual interactions with the fence within the group approaches on day two. More approaches were expected on day two as feed availability would have declined in the grazing zone and the higher feed availability in the exclusion zone may have become more attractive. However even with the increased motivation to access fresh pasture, sheep in the $100 \% \mathrm{VF}$ and $66 \%$ VF were still successfully contained by the virtual fence.

The use of manual training collars is labour intensive and limits the number of animals that can be controlled within a group as well as the number of groups able to be tested simultaneously. While, it is possible that the small number of animals used in this study may have impacted the sheep behaviour and flock movements (Arnold 1977; Taylor et al. 2011), sheep are able to display normal grazing behaviours in a minimum group of three (Penning et al. 1993). Therefore, the study size of nine animals per groups is most likely adequate in relation to expression patterns of behaviour. In the current study the split of animals that did and did not have the virtual fence implemented meant that there were three animals in the $33 \%$ VF group that had the virtual fence and three in the $66 \%$ VF without, leaving the main portion of the flock containing six animals. It is possible that the larger group of six had more influence over the three animals. In the study by Jouven et al. (2012), they worked with flock sizes of 32 and their largest split of trained animals to naïve was 50:50 leaving them with much larger groups of 16, this would be a big enough group to create a sub flock, which was observed with 13 naïve ewes spending time in their exclusion zone.

In this study, stress induced hyperthermia was not observed, and no significant differences were observed in body temperature parameters for the control and $100 \%$ virtual fenced animals, however small differences were found between $33 \%$ and $66 \%$ virtual fence animals across four of the seven parameters analysed. Temperatures circadian rhythm is described using several parameters, these are the mesor, peak, trough, range of oscillation, amplitude and acrophase, all of which were used in this study. These measures have been used previously to determine patterns in sheep temperature (D'Alterio et al. 2012). The differences observed in these measures of circadian rhythmicity indicate that there was a difference in the pattern of change for body temperature across the day between the $33 \% \mathrm{VF}$ and $66 \% \mathrm{VF}$ groups. The results observed in the $66 \%$ animals and between the control and the $100 \% \mathrm{VF}$ group suggest that exposure to the virtual fence and training animals was not inherently stressful, however further investigation is needed to determine whether exposing only a proportion of the flock will negatively impact sheep welfare in the long term. Previous work looking at sheep's initial exposure to the virtual fence electrical stimuli found that the implementation of the electrical stimulus was not highly aversive (Kearton et al. 2019). Other work has highlighted the importance of animals learning the audio cue in virtual fencing system in order to maintain good welfare through predictability and controllability (Lee et al. 2018; Marini et al. 2019). However, lack of longer-term trials to date means that the effect of repeated exposure is still to be fully tested with sheep. 


\section{8}

429

430

431

432

433 434 435

Behavioural patterns were different between the groups in this study and differed between the days of testing. Due to design limitations the study was not able to be replicated and the groups required testing on different days adding in additional environmental factors that may have influenced patterns of behaviour. The behaviours of the sheep may also have been affected by the initial adaptation period to the VF. Other studies that have looked at implementing virtual fencing in sheep have not looked at the implementation of the technology over a long period of time and have not been able to look into impacts on normal behaviour (Brunberg et al. 2017; Brunberg et al. 2015; Jouven et al. 2012). In our previous study (Marini et al. 2018a), it was found that behavioural time budgets were affected after the virtual fence was removed, through a decrease in lying behaviour, but not during the fence implementation. Studies with cattle have also found that the implementation of the virtual fence had limited impact on behavioural time budgets in cattle (Campbell et al. 2017; Campbell et al. 2019). The differences in behaviour between the two days of testing and between the groups in this study could have been due to either the short period of time that the sheep spent in the paddocks (which included no acclimation period). Or it could have been due to the restriction and reduction in pasture availability over the time-period with animals in the $100 \%$ and $66 \%$ group only accessing the inclusion zone. Animals in the control and $33 \%$ VF group accessed the entire paddock giving them more opportunity to walk and graze more pasture, which was reflected in the difference in lying time in the $33 \%$ group.

\section{Conclusions}

This study demonstrates that social influences can affect behavioural patterns and the effectiveness of virtual fencing in sheep. Only having a small portion of the flock with an active virtual fence made the fence ineffective with a much higher proportion of sheep moving forward and entering the exclusion zone, rather than turning in response to the audio cue. There were also indications of potentially negative welfare implications for the animals that were exposed to the virtual fence in the $33 \%$ VF group due to the increase proportion in electrical stimulus received. The differences seen in the behavioural patterns may have been due to sheep adapting to the system, as they were only exposed for a short period of time or potential differing environmental conditions due to testing on different days. Further studies using larger group sizes and conducted over longer periods of time are needed to confirm these findings.

\section{Acknowledgements}

This project is supported by funding from the Australian Government Department of Agriculture, Water and the Environment and CSIRO. The experiment was conducted in collaboration with Allen Buckley and Moodie Agronomy from Mallee Sustainable Farming. We thank Jim Lea, Troy Kalinowski, Damian Mowat for their technical assistance.

\section{References}


436

437

438

439

440

441

442

443

444

445

446

447

448

449

450

451

452

453

454

455

456

457

458

459

460

461

462

463

464

465

466

467

468

469

470

471

472

473

474

475

476

477

478

479

480

Arnold GW. 1977. An analysis of spatial leadership in a small field in a small flock of sheep. Applied Animal Ethology 3:263-270. https://doi.org/10.1016/0304-3762(77)90007-4

Beyer, H.L., 2004. Hawth's Analysis Tools for ArcGIS. (http://www.spatialecology.com/)

Brunberg EI, Bergslid IK, Bøe KE, and Sørheim KM. 2017. The ability of ewes with lambs to learn a virtual fencing system. Animal 11:2045-2050. 10.1017/s1751731117000891

Brunberg EI, Bøe KE, and Sørheim KM. 2015. Testing a new virtual fencing system on sheep. Acta Agriculturae Scandinavica, Section A - Animal Science 65:168-175. 10.1080/09064702.2015.1128478

Campbell D, Lea J, Farrer W, Haynes S, and Lee C. 2017. Tech-Savvy Beef Cattle? How Heifers Respond to Moving Virtual Fence Lines. Animals 7:72.

Campbell DLM, Lea JM, Haynes SJ, Farrer WJ, Leigh-Lancaster CJ, and Lee C. 2018. Virtual fencing of cattle using an automated collar in a feed attractant trial. Applied Animal Behaviour Science 200:71-77. 10.1016/j.applanim.2017.12.002

Campbell DLM, Lea JM, Keshavarzi H, and Lee C. 2019. Virtual Fencing Is Comparable to Electric Tape Fencing for Cattle Behavior and Welfare. Frontiers in Veterinary Science 6. 10.3389 /fvets. 2019.00445

D'Alterio G, Casella S, Panzera M, Gatto M, Gianesella M, Morgante M, and Piccione G. 2012. Pattern of rectal temperature in sheep affected by foot rot. Acta Scientiae Veterinariae 40:1-5.

Fisher AD, Roberts N, Bluett SJ, Verkerk GA, and Matthews LR. 2008. Effects of shade provision on the behaviour, body temperature and milk production of grazing dairy cows during a New Zealand summer. New Zealand Journal of Agricultural Research 51:99105. 10.1080/00288230809510439

Hauschildt V, and Gerken M. 2015. Individual gregariousness predicts behavioural synchronization in a foraging herbivore, the sheep (Ovis aries). Behavioural Processes 113:110-112. https://doi.org/10.1016/j.beproc.2015.01.013

Hauschildt V, and Gerken M. 2016. Effect of pasture size on behavioural synchronization and spacing in German Blackface ewes (Ovis aries). Behavioural Processes 124:15-22. https://doi.org/10.1016/j.beproc.2015.12.001

Jouven M, Leroy H, Ickowicz A, and Lapeyronie P. 2012. Can virtual fences be used to control grazing sheep? The Rangeland Journal 34:111-123. https://doi.org/10.1071/RJ11044

Kearton T, Marini D, Cowley F, Belson S, and Lee C. 2019. The Effect of Virtual Fencing Stimuli on Stress Responses and Behavior in Sheep. Animals 9:30.

Keshavarzi H, Lee C, Lea J, and Campbell DL. 2020. Virtual fence responses are socially facilitated in beef cattle. Frontiers in Veterinary Science. In Press doi: 10.3389 /fvets. 2020.543158

Lea JM, Niemeyer DDO, Reed MT, Fisher AD, and Ferguson DM. 2008. Development and validation of a simple technique for logging body temperature in free-ranging cattle. Australian Journal of Experimental Agriculture 48:741-745. https://doi.org/10.1071/EA07422

Lee C, Colditz I, and Campbell D. 2018. A Framework to Assess the Impact of New Animal Management Technologies on Welfare: A Case Study of Virtual Fencing. Frontiers in Veterinary Science 5. 10.3389/fvets.2018.00187

Marini D, Cowley F, Belson S, and Lee C. 2019. The importance of an audio cue warning in training sheep to a virtual fence and differences in learning when tested individually or in

Peer) reviewing PDF | (2020:05:48487:2:0:NEW 2 Sep 2020) 
481

482

483

484

485

486

487

488

489

490

491

492

493

494

495

496

497

498

499

500

501

502

503

504

505

506

507

508

509

510

511

small groups. Applied Animal Behaviour Science 221:104862.

https://doi.org/10.1016/j.applanim.2019.104862

Marini D, Llewellyn R, Belson S, and Lee C. 2018a. Controlling Within-Field Sheep Movement Using Virtual Fencing. Animals 8:31.

Marini D, Meuleman M, Belson S, Rodenburg T, Llewellyn R, and Lee C. 2018b. Developing an Ethically Acceptable Virtual Fencing System for Sheep. Animals 8:33.

Pedernera-Romano C, Ruiz de la Torre JL, Badiella L, and Manteca X. 2010. Effect of perphenazine enanthate on open-field test behaviour and stress-induced hyperthermia in domestic sheep. Pharmacology Biochemistry and Behavior 94:329-332. https://doi.org/10.1016/j.pbb.2009.09.013

Penning PD, Parsons AJ, Newman JA, Orr RJ, and Harvey A. 1993. The effects of group size on grazing time in sheep. Applied Animal Behaviour Science 37:101-109. https://doi.org/10.1016/0168-1591(93)90103-V

Pillot MH, Gautrais J, Gouello J, Michelena P, sibbald A, and Bon R. 2010. Moving together: Incidental leaders and naïve followers. Behavioural Processes 83:235-241. https://doi.org/10.1016/j.beproc.2009.11.006

Pinheiro J, Bates D, DebRoy S, Sarkar D, and Team RC. 2018. nlme: Linear and Nonlinear Mixed Effects Models.

Ramseyer A, Boissy A, Dumont B, and Thierry B. 2009a. Decision making in group departures of sheep is a continuous process. Animal Behaviour 78:71-78. https://doi.org/10.1016/j.anbehav.2009.03.017

Ramseyer A, Boissy A, Thierry B, and Dumont B. 2009b. Individual and social determinants of spontaneous group movements in cattle and sheep. Animal 3:1319-1326.

Sanger ME, Doyle RE, Hinch GN, and Lee C. 2011. Sheep exhibit a positive judgement bias and stress-induced hyperthermia following shearing. Applied Animal Behaviour Science 131:94-103. https://doi.org/10.1016/i.applanim.2011.02.001

Taylor DB, Price IR, Brown WY, and Hinch GN. 2011. Effects of Merino flock size, paddock complexity and time of day on response to trained leaders. Small Ruminant Research 97:35-40. https://doi.org/10.1016/j.smallrumres.2011.01.010 
Figure 1

Schematic of the experimental setup, the dotted line indicates the virtual fence position.

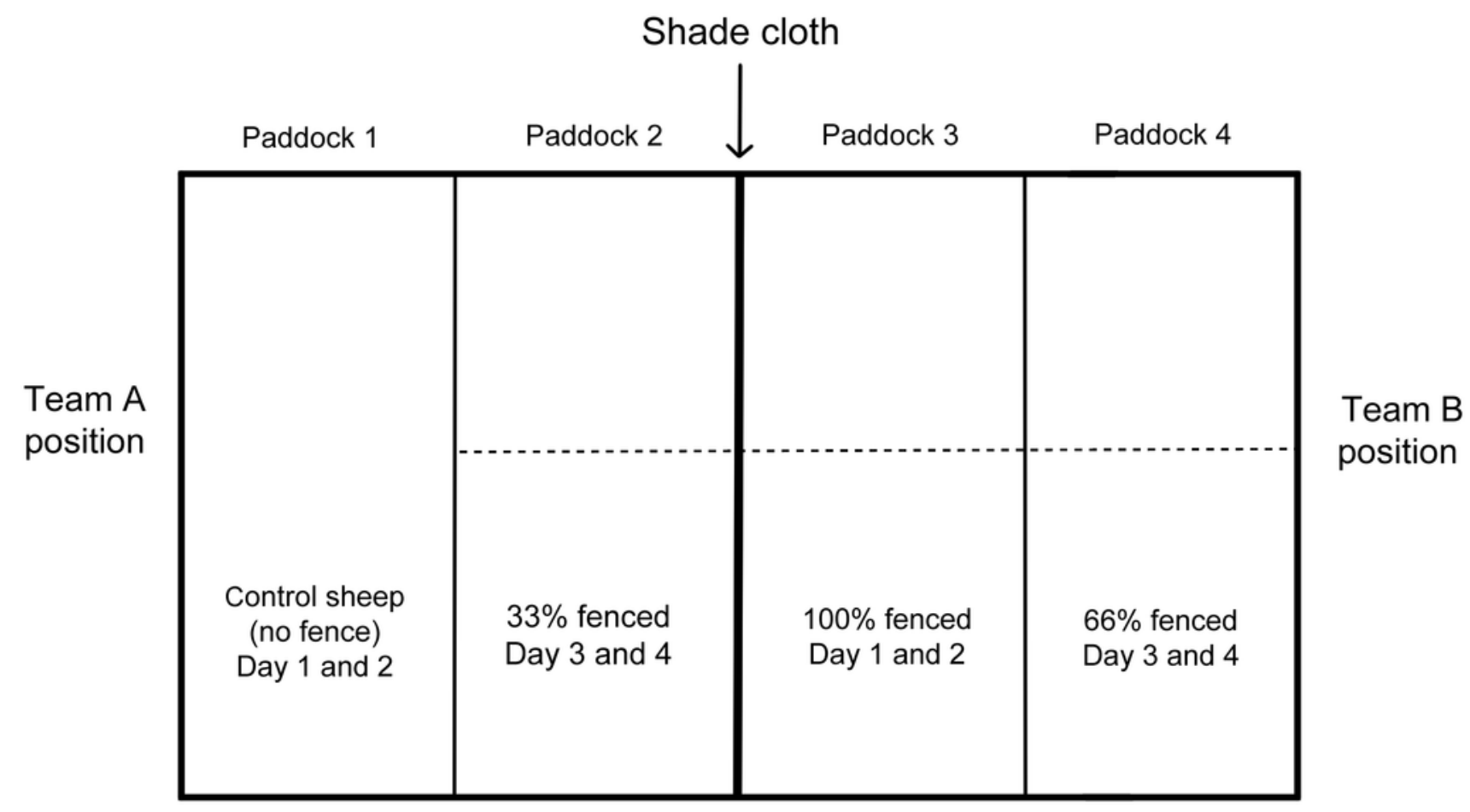


Figure 2

Residency maps for the flock in each group ( $\mathrm{n}=9$ sheep each) at Waikerie showing successful exclusion from northern zone under $100 \%$ and $66 \%$ active virtual fence groups.

Each cell indicates a grid of 2 meters, colour indicates the time spent in each cell (mins). The darker the cell colour the more time spent.

Waikerie virtual fence residency grids

Control

13th \& 14th March

$33 \%$ Collared

15th \& 16th March

$100 \%$ Collared

13th \& 14th March

$66 \%$ Collared

15 th \& 16 th March
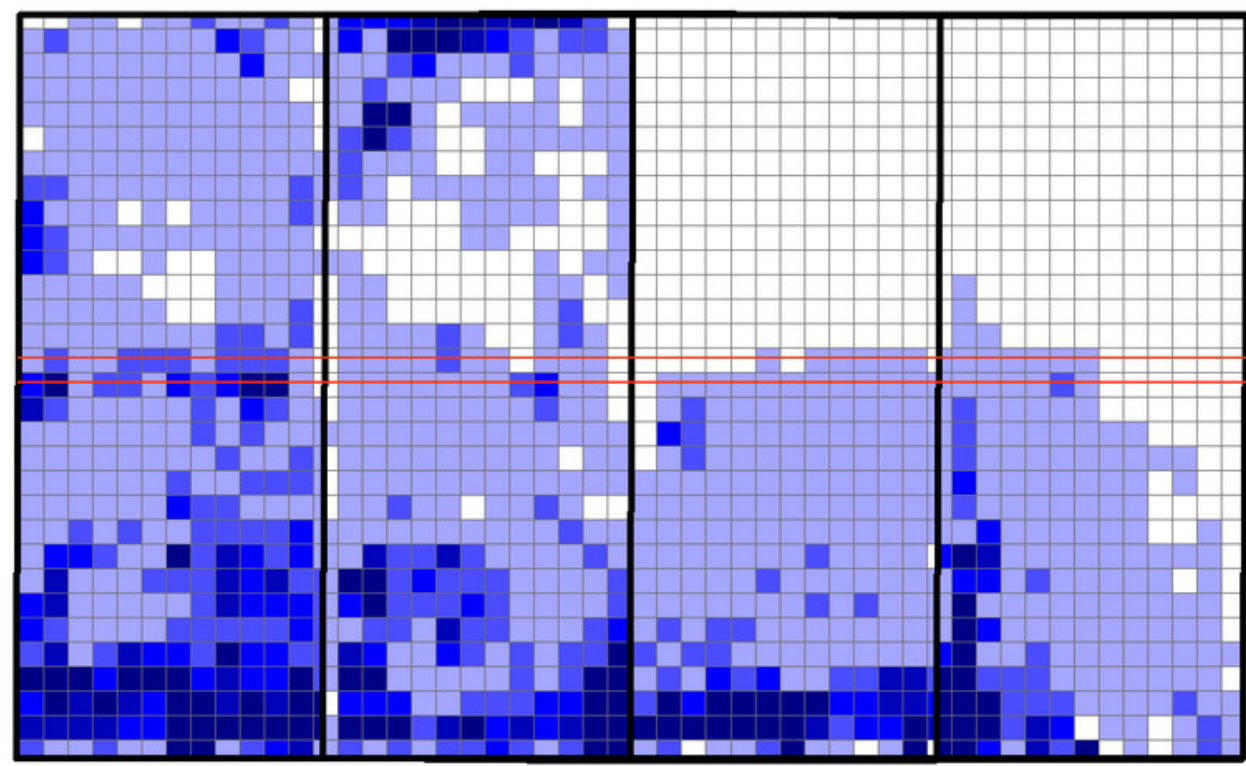

Time spent in area (mins)

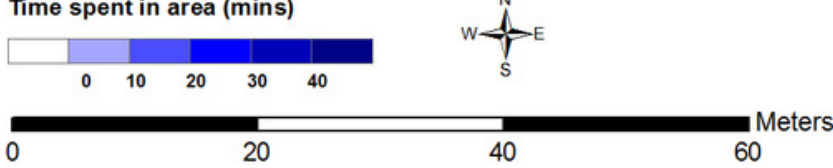


Figure 3

Proportion of time spent displaying standing, lying or walking behaviours for each group on day 1 and day 2 of the virtual fencing testing

The solid black shading is time spent walking, solid grey shading is time spent lying, horizontal lines indicate time spent standing. Time is displayed as a proportion (\%) of total activity recorded for each day. 


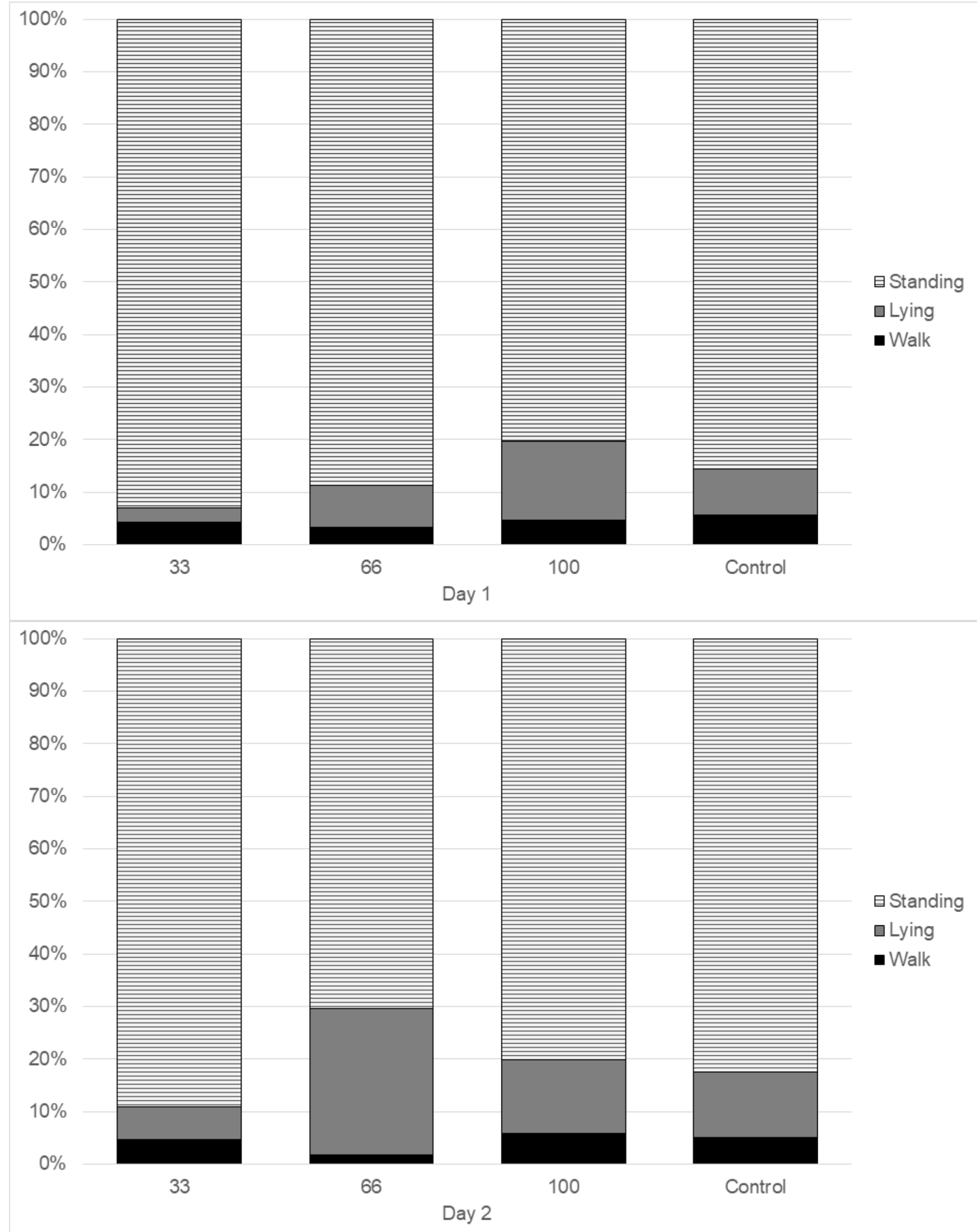




\section{Table $\mathbf{1}$ (on next page)}

Summary of total number of group approaches and total number of audio cues and electrical stimuli given to individuals on interaction with the virtual fence. Data presented for groups that had the virtual fence implemented. 
2 Table 1: Summary of total number of group approaches and total number of audio cues and electrical stimuli given to individuals on interaction with the virtual fence. Data presented for groups that had the virtual fence implemented.

\begin{tabular}{c|ccc|ccc}
\multicolumn{9}{c}{ Day 1 } & \multicolumn{2}{c}{ Day 2 } \\
\hline $\begin{array}{c}\text { Group } \\
\text { (\% with fence) }\end{array}$ & $\begin{array}{c}\text { Group } \\
\text { Approaches }\end{array}$ & Audios & Elec Stim & $\begin{array}{c}\text { Group } \\
\text { Approaches }\end{array}$ & Audios \\
33 & 4 & 14 & 5 & 9 & 47 & 26 \\
66 & 3 & 15 & 5 & 5 & 25 & 56 \\
100 & 12 & 17 & 3 & 12 & 13
\end{tabular}




\section{Table 2 (on next page)}

Count of behaviours displayed in response to the audio and electrical cues from sheep in the $33 \%, 66 \%$ and $100 \%$ virtual fence groups over the two days the virtual fence was implemented. 


\begin{tabular}{|c|c|c|c|}
\hline Response to audio & $33 \%$ & $66 \%$ & $100 \%$ \\
\hline Grazing & 17 & 13 & 35 \\
\hline Stop/lifted head & 10 & 9 & 16 \\
\hline Turn & 7 & 10 & 20 \\
\hline Walk forward & 23 & 8 & 2 \\
\hline Run forward & 4 & 0 & 0 \\
\hline Total interactions & 61 & 39 & 73 \\
\hline
\end{tabular}

1 Table 2: Count of behaviours displayed in response to the audio and electrical cues from sheep in the $33 \%, 66 \%$ and $100 \%$ virtual

2 fence groups over the two days the virtual fence was implemented. 


\section{Table 3 (on next page)}

Count of behaviours displayed in response to the audio and electrical cues from sheep in the $33 \%, 66 \%$ and $100 \%$ virtual fence groups over the two days the virtual fence was implemented. 
Table 3: Count of behaviours displayed in response to the audio and electrical cues from sheep in the 33\%, $66 \%$ and $100 \%$ virtual fence groups over the two days the virtual fence was implemented.

\begin{tabular}{r|ccc} 
Response to electrical stimulus & $\mathbf{3 3 \%}$ & $\mathbf{6 6 \%}$ & $\mathbf{1 0 0 \%}$ \\
\hline No response & 3 & 0 & 0 \\
Turned & 3 & 1 & 7 \\
Jumped and turn & 5 & 1 & 1 \\
Run/jump forward & 5 & 0 & 0 \\
Hop forward & 15 & 11 & 15
\end{tabular}




\section{Table 4 (on next page)}

Body temperature parameters over the two days of testing for all groups

Temperature presented as mean degrees ${ }^{\circ} \mathrm{C}+$ - S.E.M. 
Table 4: Body temperature parameters over the two days of testing for all groups

\begin{tabular}{|c|c|c|c|c|c|}
\hline \multirow[b]{3}{*}{ Fitted curve parameters } & \multicolumn{5}{|c|}{ Group } \\
\hline & Control & $100 \%$ collared & $33 \%$ collared & $66 \%$ collared & \\
\hline & Mean \pm s.e.m. & Mean \pm s.e.m. & Mean \pm s.e.m. & Mean \pm s.e.m. & \\
\hline Mesor & $39.0 \pm 0.14$ & $39.1 \pm 0.12$ & $39.0 \pm 0.07$ & $39.3 \pm 0.08$ & n.s \\
\hline Peak & $39.5 \pm 0.17$ & $39.7 \pm 0.13$ & $39.9 \pm 0.07$ & $39.9 \pm 0.12$ & n.s. \\
\hline Trough & $38.4 \pm 0.11$ & $38.6 \pm 0.10$ & $38.4 \pm 0.11$ & $38.8 \pm 0.09$ & 0.002 \\
\hline Range of oscillation & $1.1 \pm 0.09$ & $1.1 \pm 0.10$ & $1.5 \pm 0.08$ & $1.1 \pm 0.09$ & 0.001 \\
\hline Amplitude & $0.4 \pm 0.04$ & $0.6 \pm 0.05$ & $0.9 \pm 0.05$ & $0.6 \pm 0.09$ & 0.006 \\
\hline Acrophase & $17.7 \pm 0.78$ & $16.2 \pm 1.16$ & $12.9 \pm 1.17$ & $15.9 \pm 0.17$ & 0.03 \\
\hline$S D$ & $0.3 \pm 0.02$ & $0.3 \pm 0.03$ & $0.3 \pm 0.03$ & $0.3 \pm 0.02$ & n.s. \\
\hline
\end{tabular}

